\title{
Computational electromagnetics for design optimisation: the state of the art and conjectures for the future
}

\author{
J.K. SYKULSKI* \\ School of Electronics and Computer Science, University of Southampton, Southampton SO17 1BJ, United Kingdom
}

\begin{abstract}
The paper reviews the state of the art in modern field simulation techniques available to assist in the design and performance prediction of electromechanical and electromagnetic devices. Commercial software packages, usually exploiting finite element and/or related techniques, provide advanced and reliable tools for every-day use in the design office. At the same time Computational Electromagnetics continues to be a thriving area of research with emerging new techniques and methods, in particular for multi-physics applications and in the area of multi-objective optimisation.
\end{abstract}

Key words: computational electromagnetics, CAD in magnetics, design and optimisation, electromagnetic fields.

\section{Introduction}

Designers of modern electrical equipment need to satisfy customers and be competitive in terms of low first and operating costs, high efficiency, reliability, minimum weight, demanding tolerances, etc. Moreover, new types of devices and new materials are being developed and implemented. Thus it becomes increasingly critical to analyse any proposed design in considerable detail, so that a near optimum may be achieved.

Recent advances in Computational Electromagnetics, supported and encouraged by continually increasing power and speed of computers, make finite element and related techniques an attractive alternative to established approximate and empirical design methods, including the still popular 'trial and error' approach. Numerical field analysis has undergone tremendous transformation over the years resulting in reliable computer codes for design purposes. There are several specialised conferences and symposia dedicated to development of methods and simulation techniques for magnetic, electric and electromagnetic field computation, the two major being COMPUMAG [1] (organised by the International Compumag Society [2]) and CEFC [3] (sponsored by the IEEE Magnetics Society), both reporting on progress in theory and software methodology in the context of real engineering applications at low and high frequencies. There are other focused meetings like CEM (Computation in Electromagnetics), organised by the Professional Network on Electromagnetics of the IET (Institution of Engineering and Technology, London) with selected papers published as a special issue of IEE Proceedings [4] and now IET Science Measurement and Technology [5]; ISEF (International Symposium on Electromagnetic Fields in Electrical Engineering) [6]; EPNC (Symposium on Electromagnetic Phenomena in Nonlinear Circuits) [7] and others. Application oriented conferences such as ICEM (International Conference on Electrical Machines) often have an appreciable proportion of papers devoted to field computa- tion techniques and a section dedicated to finite element modelling [8]. Specialist meetings discussing optimisation are also very relevant [9].

The research effort of the Computational Electromagnetics community is overseen and coordinated by the already mentioned International Compumag Society [2], an independent organisation uniting around 700 members from over 40 countries, which has as its mission the advancement and dissemination of knowledge about the application of computer methods to field problems having significant electric, magnetic or electromagnetic components. The ICS Newsletter [10] publishes regular review articles on 'hot topics' in electromagnetics, almost always with strong applications emphasis and often with design focus. Another form of networking is offered by the IET via its Knowledge Network on Electromagnetics [11].

There are many books introducing the 'art' and 'craft' of field computation to practicing engineers and designers; these range from fundamental textbooks [12] to advanced monographs [13-15]; some are very specifically related to electrical power engineering in general [16] or design of electrical machines in particular [17]. Books on CAD in magnetics are also available [18], although there is a certain shortage of a more modern treatment of the topic. Overall, there is a vast literature on the subject covering various aspects of field simulations in the context of design and performance prediction of electrical devices. Many methods are available and commercial software packages offer efficient modelling and simulation tools. However, there is also a certain aura of mysticism surrounding the subject.

\section{The industrial perspective}

Computational Electromagnetics (CEM), that is the procedures for approximating electromagnetic fields by means of numerical algorithms, has become a mature subject - while remaining an active research discipline in its own right - prac-

*e-mail: jks@soton.ac.uk 
tised by a large international community serving science and industry. Computer modelling is used at all design stages and it is generally acknowledged that the sole use of analytical and experimental methods, followed by expensive and inflexible prototyping, is no longer cost-effective. However, it is perhaps also true to say that many industrialists - the very people who could benefit most by introducing field modelling software to cut design times and costs - still perceive CEM as a kind of "black magic". At the same time, since research funding available for fundamental work in this field is scarce, a direct involvement of industry is increasingly becoming a necessity. But benefits have to be demonstrated to managers before they commit resources to support fundamental developments. All this may sound only too familiar to researchers struggling to secure funding, but there is a message to the community to be more proactive in promoting CEM as an efficient design tool.

Closely related to industrial are educational needs, which are often driven by different practical requirements. It could be argued that three levels of skills may be required in the design office:

1. a majority of users who should be able to run specialist electromagnetic software confidently, understand field displays, process numerical results and incorporate them into the design;

2. design 'experts' who will understand the language of electromagnetics and are able to create computational models using available commercial software;

3. electromagnetic software developers - the ultimate CEM experts creating computational tools to be used for design purposes.

In the early days researchers regarded writing computer programs as a 'cultural extension' to their work and there was often a free exchange of codes. Obviously this is no longer rational as real costs are involved and software production has become a commercial activity. There is no fundamental difference between hardware and software in this respect, as both require development, maintenance and support.

Electromagnetic products permeate modern life and it is often taken for granted that the designers have achieved the best performance at least cost. Unfortunately, finding the best size, shape and characteristics for the components - even using the best simulators available - may be time consuming and costly, and hence likely to be incomplete. There are delays in bringing improved products to market and opportunities for even better products are often missed. However, making the subject more appealing to both managers and students appears to be the crux.

\section{Commercial software}

It is not the intention of this section to provide a comprehensive catalogue of available electromagnetic software. Notwithstanding, it appears worthwhile to mention at least some commercially available systems offering integrated tools for CAD in magnetics. A typical commercial package will include most of the following components:
- Pre- and Post-Processing: fully interactive via dedicated user interface, advanced viewing facilities, a range of supported output devices, automatic and (increasingly) adaptive meshing;

- Statics: magneto- and electrostatic analysis using nonlinear (and often anisotropic and hysteretic) materials, modelling of permanent magnets, special versions for laminated structures;

- Quasi-static: steady-state (single frequency ac) eddycurrent analysis, incorporating complex permeability, approximate non-linear solutions (fundamental harmonic field), background dc fields, voltage-driven formulations;

- Transient eddy currents: full transient time simulations, non-linear materials, multiple drives and background dc fields;

- Motional eddy currents: motion induced eddy-current analysis (with constant or varying topology);

- Stress and thermal: mechanical stress or thermal analysis using forces or ohmic heating losses, respectively, calculated from electromagnetic solutions;

- 2D, 2D axi-symmetric and $3 D$ formulations.

The following is a non-exhaustive list, with relevant web links, of the most popular software packages already in extensive use in design offices:

- OPERA, Vector Fields Ltd [19];

- MagNet, Infolytica [20];

- Maxwell, Ansoft [21];

- ANSYS [22];

- FLUX, CEDRAT Software [23];

- MEGA [24];

- Integrated Engineering Software [25].

Moreover, there are many 'in-house' systems developed by academic or research institutions, some commercially available. Finally, there exists software written specifically for particular devices, such as SPEED [26] for designing electrical machines, which can link to the general purpose finite element packages listed above.

\section{Pioneering developments in CEM}

A fairly recent comprehensive survey of the key developments in CEM and their attribution will be found in [27]. It may be helpful to recall some of the main achievements and milestone contributions to the art of numerical field computation. In fact many of the ground rules can be traced back to Southwell and his work using Finite Differences (FD) as long ago as in the 1940s [28]. The Finite Element method (FE) grew out of structural mechanics in applications to aircraft industry ' [29], and the developments were strongly driven by the needs of the relevant industries; it was not until much later that the method was studied by mathematicians. A significant milestone, as far as electromagnetic field is concerned, occurred in 1963 when Winslow [30] reported on a discretisation scheme based on an irregular grid of plane triangles. He used a generalised finite difference scheme but also introduced a variational principle, both giving the same results. 
The latter approach may be viewed as equivalent to the FE method and is consequently the earliest example of this technique in electromagnetics. Silvester and co-workers at McGill University advanced the formulation using unstructured meshes and higher order elements. The polynomials proposed by Silvester [31] using simplex coordinates allowed most formulations to be accomplished for a prototypal triangle. 1970 saw the first application of the method to rotational electrical machines by Chari and Silvester [32].

In the 1970 s the CEM community was effectively created when researchers in academia, national laboratories and industry started to exchange ideas. The breakthrough occurred in 1976 when the first Compumag Conference was held in Oxford. Several developments were reported leading to significant advances in theory, formulations, numerical techniques and algorithms. For example, the Incomplete Cholesky Conjugate Gradient method (ICCG) was introduced for solving large sparse systems of equations [33, 34], where the operation count is approximately $n \log n$ and largely independent of the bandwidth; the algorithm is still the root of most contemporary codes. Another significant advancement was in the now prevalent utilisation of the 'Delaunay meshing', with the original idea going back to 1934 and efficient algorithms implemented more recently in 2D [35] and 3D (using tetrahedral elements) [36] including error analysis.

It was also suggested to use Kelvin Transformation, where the exterior space to a sphere in 3D (or circle in 2D) surrounding the actual model may be solved as an interior problem [37, 38], as a way to model boundaries at infinity. Another milestone development was the introduction of 'Edge Elements' and differential forms. Known more generally as 'Whitney forms' these elements were first presented to the CEM community by Bossavit [39, 40], followed by Biro et al. [41] and Tsibouikis et al. [42]. It is also argued that, in comparison to the vector calculus description, differential forms make electromagnetism simpler, clearer, and more intuitive $[43,44]$ as the relationships may be illustrated by simple diagrams [45]. The importance of constitutive equations is highlighted as they are shown to associate energy density with infinitesimal volumes and therefore energy with complete systems. This gives rise to dual energy formulations, as demonstrated by Hammond [46], and a numerical technique known as 'tubes and slices' [47].

Of crucial importance and interest to designers of modern electrical equipment is modelling of properties of materials, in particular magnetic hysteresis and anisotropy. Various formulations have been developed of which the most widely used are scalar and vector Preisach models; the fundamental work was undertaken by Mayergoyz [48], while a comprehensive review of modelling techniques may be found in [49]. Furthermore, new types of materials have been developed in recent years which require novel models. For example, soft magnetic composites made from powder [50] continue to have an impact. Possible benefits include faster manufacturing at lower cost, improved thermal properties and higher frequency capability. Another exciting new family of materials is high temperature superconductors, which offer great potential in terms of in- creasing efficiency of devices and/or reducing their size. However, they present a considerable modelling challenge because of very high non-linearity and anisotropic properties [51, 52].

Another challenging problem is application of FE to systems under dynamic conditions, as inevitably some form of moving meshes is required. Several elegant solutions have been developed, including special air-gap elements coupling analytical solutions for the air-gap with a standard FE formulation [53], the utilisation of Lagrange multipliers to couple independent FE meshes which are free to rotate [54], overlapping meshes [55] and moving band techniques [56].

Finally, although finite elements have proven to be the most flexible technique to model practical engineering devices and systems, other methods also exist with reported successful implementations. The Transmission Line Matrix (TLM) method [57, 58] should be mentioned here as well as the whole family of formulations based on finite integration (see for example [59]). Of particular importance is the Boundary Element Method (BEM) [60], often favoured as only surfaces need to be meshed making the codes easier to use. However, non-linearity and skin effect may be an issue so hybrid FE-BEM formulations have also been developed [61].

\section{The state of the art}

As a result of successful implementation of new techniques, new efficient, accurate and numerically stable algorithms have emerged. The following is a subjective non-prioritised list of recent advances which have made the greatest impact on the CEM community:

- higher order Finite Difference Time Domain (FDTD),

- the Multiple Multipole Technique (MMT),

- further developments of the Transmission Line Matrix (TLM) methods,

- a new Finite Element Difference (FED) method,

- a Subspace Projection Extrapolation (SPE) scheme,

- the use of Finite Integration Technique (FIT),

- the usage of total/reduced magnetic vector potential and electric scalar potential,

- formulations in terms of differential geometry,

- implementation of edge and facet elements,

- improved anisotropy and hysteresis models,

- efficient application of Continuum Design Sensitivity Analysis (CDSA),

- multi-objective optimisation.

The main CEM conferences COMPUMAG [1] and CEFC [2], as well as the other meetings already mentioned [3-9], provide a continuing source of information about further advances. As examples, two particular areas will be mentioned, with which the author has been closely involved, namely the computation of electromagnetic forces and application and modelling of superconducting materials.

The knowledge of the total force and its distribution is often crucial in the design of electromechanical devices (e.g. electrical machines). The two most commonly used techniques are based on the Maxwell Stress Tensor (MST) and the Virtual Work Principle (VWP). MST is derived from the Lorentz 
force expression, while VWP relates the force to changes in stored energy. A thorough treatment of the principles of force formulations, as well as physical and implementation aspects, may be found in $[62,63]$. The key advantage of using MST is that only one field solution is required, but there are significant implementation problems related to practical numerical solutions (e.g. the need for a very fine mesh in air-gap regions). The VWP, on the other hand, calculates forces by applying a virtual displacement of a body and considering the associated change in the co-energy of the system. Unfortunately, the necessary gradient of the co-energy function is rarely available explicitly and thus at least two field solutions are needed, or even more to provide better accuracy. Ways of improving accuracy and reducing computational effort have been addressed by Coulomb [64], McFee [65] and Hameyer [66], amongst others. It is also worth highlighting a completely new force computation algorithm based on continuum design sensitivity analysis [67-69]. The formulation permits the sensitivity of any global quantity to a perturbation in a parameter to be computed without reference to the actual numerical computation scheme. In other words, it allows for a Virtual Work calculation without the need for a physical displacement. The resultant expressions resemble the MST but have the crucial advantage of the integration taking place on the surface of a material rather than in the air outside. The formulation allows for global forces - as well as force distributions over the surface of a body - to be computed, including the case of a zero air gap (which MST does not allow introducing a rather important limitation of the MST approach). Furthermore, the force expressions clearly demonstrate the contributions to the global force from different sources of magnetic field. Finally, the implementation is simple, independent of the numerical analysis method used and can be easily applied in combination with commercial software.

The discovery and advances in new materials present a modelling challenge and often require reformulation of fundamental equations or design methods. As an example the recent advances in superconducting technology will be addressed. Ceramic superconductors were first announced in 1986. Their main advantage is ability to operate at liquid nitrogen temperatures $(78 \mathrm{~K})$ - hence the name High Temperature Superconductors (HTS) - which makes it a relatively cheap and reliable technology (sometimes compared to water cooling). With practical current densities of possibly up to 100 times larger than in conventional (copper) windings they are considered as potentially advantageous in electric power applications (generators, motors, fault current limiters, transformers, flywheels, cables, etc.), as losses are greatly reduced and power output per volume increased. To the designers they present a challenge due to very highly non-linear characteristics and anisotropic properties, and due to unconventional design solutions. The ability to predict and reduce all 'cold' losses (that is losses released at cryogenic temperature) is of vital importance. Moreover, the HTS materials behave differently to conventional conductors. One of the first HTS devices designed, built and tested was a small demonstrator transformer [70]. A particularly satisfying result was a two-fold reduction of losses accomplished by insertion of magnetic flux diverters reducing an unwanted component of magnetic field in the coil region. Some more general discussion pertaining to large HTS power transformers may be found in [71]. Another successfully completed design was of a synchronous generator [72]; in terms of modelling the key issues were no-load tooth ripple losses (due to the distortion of the fundamental flux density wave by the stator slotting) and full-load losses (which include the effects of the MMF harmonics of the stator winding). The field penetration into the HTS tape was shown to be accurately simulated using various diffusion models [73, 74]. Another challenge was to reduce the harmonic content in the MMF wave [75]. Conceptual alternative designs are discussed in [76].

Continuing progress in CEM methods is required and currently undertaken research includes: efficient handling of nonlinearity, hysteresis and anisotropy; adaptive meshing and reliable error estimation; modelling of linear movement and rotation of some parts of the device; combined modelling of fields and supplying electronic circuitry; coupled and multi-physics problems; and finally integrated design systems. Finally, helpful correspondence between field and circuit descriptions can be established and has been explored in [77-80].

\section{Computer Aided Design}

The CEM assisted Computer Aided Design (CAD) has come of age in the electromechanical and electromagnetic industry. However, difficulties are experienced by designers, especially when first introduced to the subject. It could be argued that these difficulties arise in two areas: (i) inadequate understanding of relevant electromagnetic theory, and (ii) inability to appreciate the intricacies of numerical modelling. Thus the importance of engineering judgement becomes paramount. There is a risk of users having too much 'confidence' in the results of simulations with no proper consideration regarding the validity of assumptions and modelling simplifications. To put it simply, the answer can only be as good as the model adopted. A useful 'check list' of questions (based on [16]) which need to be addressed by users attempting to use CAD systems for electromagnetic design could include the following:

- Is a 2D model adequate?

- If so, is it necessary to allow for 'end effects'?

- If 3D is essential, what simplifications can be made?

- What is the most appropriate potential to use?

- How much of the surroundings need to be modelled?

- Do symmetry and/or periodicity conditions exist?

- What other boundary conditions can be assumed?

- Must induced currents be allowed for?

- If so, what is the highest frequency to be considered?

- Are materials non-linear, anisotropic, hysteretic?

- Are all material characteristics available and accurate?

- Which critical areas require fine discretisation?

- Are variants of the base design to be investigated?

- Can second-order effects be neglected?

- Is supplying circuit necessary in the model?

- What quantities are required from the solution? 
Clearly the above list could be extended almost indefinitely, but it does stress the importance and central role of the designer in the process, someone taking full responsibility for the outcome who is much more then an 'operator' for launching the software. However, a well constructed CAD system will offer as much automation as feasible (and practical) to allow the designer to focus on the main task at hand rather then concerning himself with the details of how to run the software. Finally, ideally a successful design of an electromechanical device should be optimised; this introduces an additional challenge to software producers, as optimal design usually necessitates repetitive usage of finite-element or other numerically intensive field computation solver.

A simple (but 'expensive') way of incorporating field modelling into an optimisation loop is to run the FE package every time an objective function evaluation is required. Although straightforward in implementation, this 'on-line' approach would normally lead to unacceptable computing times, as for each set of selected design parameters a full field analysis may need to be performed. The number of necessary FE calls escalates as the number of design variables increases; moreover, additional FE runs may be needed to calculate gradients of the objective function. Although theoretically this is of no consequence, in the engineering design office environment such an approach becomes impractical. Thus significant effort is currently directed at developing optimisation techniques suitable for such computationally intensive problems [81, 82]. One recent method, which has attracted significant attention, is called surrogate modelling, a functional relationship between the design variable space and the objective function space constructed based on design vectors which have their objective function values known. A type of surrogate model known as kriging appears to be particularly useful [83].

Design has to be considered in the context of general trends in optimisation methods. The importance of multiobjective tasks is increasing as practical designs usually involve conflicting requirements. Such problems may be converted into single-objective tasks with a priori application of knowledge or imposition of a decision (e.g. weighting factors), but it is argued that information can easily be lost in the process. Instead the application of Pareto Optimal Front is advocated. A survey of recent advances in optimisation is provided in Sec. 7.

Finally, from an engineering point of view, it is often an improvement to the design, not necessarily a global optimum, which is sought. Hence the sensitivity analysis is of great value as computing times are not affected by the number of design variables. The Continuum Design Sensitivity Analysis (CDSA) is particularly to be recommended as standard EM software may be used for extracting gradient information [84-86].

\section{New trends in optimisation}

Optimal electromagnetic design is an area of vigorous research involving mathematics, numerical analysis, software development and engineering design. A peculiarity of electromagnetic design, as argued above, is that the solutions are 'expensive' and thus 'cost-effective algorithms' have to be used. The following section is based on the author's recent publication [87].

7.1. 'No free lunch' theorem. The 'no free lunch' (NFL) theorem [88] prohibits the existence of an algorithm which would outperform all other optimisation algorithms, when averaged over all possible problems. It argues that, averaged over all problems, every algorithm performs the same. However, as design engineers are only interested in a subset of problems, thus - consistent with the NFL theorem - it is possible to identify a set of algorithms which outperform others over a particular domain of interest [89]. Several methods exist for achieving cost-effectiveness in multi-objective optimization, including small population genetic algorithms, hybrid algorithms, reduction of design variables and fitness inheritance. In this paper we focus on surrogate modelling and krigingassisted methods [90, 91].

7.2. Surrogate modelling and kriging. The simplest to construct and visualise are polynomial models. However, they have several shortcomings: low-order polynomials are incapable of modelling complex functions, whilst high-order ones often result in ill-conditioned matrices; the model can only be constructed after a certain number of observations; and it is only for this minimum number of observations that they are interpolating. Moreover, in order for a surrogate model to be interpolating, it is necessary to use some additional basis functions. An exponential basis function leads to the idea of kriging. First introduced half a century ago in geostatistics it has also found applications in other fields. A version for predicting experiments with deterministic output, known as Design and Analysis of Computer Experiments (DACE), was developed in the late 1980s [92].

According to Jones' taxonomy [90] two criteria are used: the type of the surrogate model and the method of selecting search points. The former is further subdivided into those that interpolate the observed points and those which do not; the latter into two-stage and one-stage varieties. In two-stage algorithms first the surrogate model is fitted to the observed points and then a utility function is constructed to determine the next search point. In one-stage methods a design vector is determined which would yield the most credible response surface.

An alternative taxonomy for kriging assisted methods provides classification according to the number of design vectors to be evaluated at each iteration and how 'tunable' each method is to the balance between exploration and exploitation. The Efficient Global Optimisation (EGO) algorithm [93] uses the concept of expected improvement, which may be viewed as a fixed compromise between exploration and exploitation. It is currently acknowledged as one of the best performing methods for single-objective optimization problems (SOOP). Non-target based tunable utility functions allow the balance between exploration and exploitation to be controlled by a parameter which is not an estimate of the global minimum. Two 
simple utility functions exist which are of this type: the Generalized Expected Improvement (GEI), and the Weighted Expected Improvement (WEI) [94]. Target based tunable utility functions, on the other hand, allow the balance between exploration and exploitation to be controlled by a parameter which is an estimate of the global minimum, while the concept of improvement is also used. Two methods have been acknowledged as most promising: the probability of improvement with multiple targets, and the one-stage credibility of hypotheses method (with either single or multiple targets at each iteration) [90, 95].

7.3. Multi-objective optimization. In a multi-objective optimization problem (MOOP) we try to simultaneously minimize different objectives, giving rise to ideal and utopian objective vectors, something which in general is not possible, leading to the necessity of defining exactly what constitutes a solution to the MOOP; the objectives are almost always in competition and the definition usually adopted is that of the Pareto-Optimal Front (POF) [96]. In the absence of other criteria, all POF solutions are equally important and as many should be found as possible. The simultaneous two goals are to find a set of solutions as close as possible to the POF and a set of solutions as diverse as possible; the latter is important because it assures us that no single objective is being favoured. Achieving a good balance between convergence to and diversity along the POF is important to all multi-objective optimization algorithms.

Multi-objective methods using surrogate models may be categorised as scalarizing and non-scalarizing; the former combine the multiple objectives of the MOOP (using a function) and use one of the methods for SOOP. By varying parameters controlling the way in which the multiple objectives are combined, an approximation to the POF can be built up, but care must be taken to ensure that the contours of the resulting function are able to capture every point on the Pareto-optimal front. The most popular methods here include: $\varepsilon$-constraint (which considers only one of the objectives for minimisation, whilst treating the other objectives as constraints to be satisfied), weighting (each objective is associated with a weighting coefficient), weighted and augmented weighted $L_{p}$, and weight$e d$ and modified weighted Tchebycheff metric (using different metrics to define the distance of a solution from the Utopian point) [96]. After transforming a MOOP to a SOOP, any method may then be used to solve the resulting SOOP. This creates a huge number of possible cost-effective MOOP algorithms. Surprisingly few have been pursued in the literature, with two notable exceptions: EGO [93] and ParEGO [97].

Non-scalarizing methods, on the other hand, consider each objective function individually, e.g. by evaluating the Pareto points predicted by the multiple surrogate models; an example is an optimisation of a switched reluctance motor reported in [98]. Many 'greedy' MOOP algorithms exist which are non-scalarizing, in particular Multi-Objective Evolutionary Algorithms; however, non-scalarizing methods for costeffective multi-objective optimization have only appeared fairly recently. In [99] and [100] the notion of 'equivalent' and 'dominating' designs is used to establish the probability of improvement utility function.

Surrogate models cannot be used to select every design vector during the search: a certain minimum need to be sampled before a kriging model can even be constructed. This initial set is called an experimental design and the theory behind selecting suitable points is known as Design of Experiments [101]. The two most common modern experimental designs are the Latin Hypercube [93] and the Hammersley sequence [102].

\section{What the future holds}

Predicting the future is like looking into a crystal ball and hardly a scientific approach, but learning from the past experiences and watching current developments is worthwhile. The premises of this article are that Computational Electromagnetics is a very active area of research, the achievements to date are considerable and the tremendous effort of the community continues. General purpose and specialised software packages offer flexible design tools while virtual prototyping increasingly becomes a norm rather than an exception. One of the challenges for the designers and practising engineers is to 'keep up' with the technology; this may be achieved by monitoring the relevant conferences and other events. With this in mind the following is a list (with web links provided in References) of recent and forthcoming meetings where further advances in CEM and their relevance to electromagnetic design have been or are likely to be discussed: CEFC [103], ISEF [104], ICEM [105], IGTE [106] and above all COMPUMAG [82].

\section{Conclusions}

This paper is an attempt to review the significant advances in the field of Computational Electromagnetics to demonstrate how numerical field simulation could aid the design of electromagnetic and electromechanical devices. Based mostly on the versatile finite element approach, the available software, including general purpose commercial packages, offer a mature tool for performance prediction, optimisation and general design. Tackling the multi-physics problems and multi-objective optimisation are identified as the biggest current challenges.

\section{REFERENCES}

[1] COMPUMAG 2007 Proceedings, IEEE Transactions on Magnetics 44 (6), (2008).

[2] International Compumag Society, http://www.compumag.co.uk/.

[3] CEFC 2006 Proceedings, IEEE Transactions on Magnetics, 43 (4), (2007).

[4] Special Issue on Computational Electromagnetics, IEE Proceedings, SMT 149 (5) (2002), and 151 (6) (2004).

[5] IET Sci. Meas. Technol. 1 (1), (2007).

[6] ISEF 2005, International Symposium on Electromagnetic Fields in Electrical Engineering, selected papers, COMPEL 27 (3), (2008).

[7] EPNC 2008, Symposium on Electromagnetic Phenomena in Nonlinear Circuits, selected papers, COMPEL 28 (3), (2009). 
[8] $18^{\text {th }}$ International Conference on Electrical Machines, 1-6 (2008).

[9] Workshop on Optimization and Inverse Problems in Electromagnetics OIPE, COMPEL 26 (2), (2007).

[10] J.K. Sykulski, International Compumag Society Newsletter, ISSN 1026-0854.

[11] Professional Network Electromagnetics, IET, London, UK, http://kn.theiet.org/communities/electromagnetics/about.cfm.

[12] P. Hammond and J.K. Sykulski, Engineering Electromagnetism, Physical Processes and Computation, Oxford Science Publications, New York, 1994.

[13] J.K. Sykulski, Computational Magnetics. Chapman \& Hall, London, 1995.

[14] R.L. Stoll, The Analysis of Eddy Currents, Clarendon Press, Oxford, 1974

[15] K.J. Binns, P.J. Lawrenson and C.W. Trowbridge, The Analytical and Numerical Solution of Electric and Magnetic Fields, John Wiley \& Sons, New York, 1992.

[16] A.B.J. Reece and T.W. Preston, Finite Element Methods in Electrical Power Engineering, Oxford Science Publications, Oxford, 2000.

[17] K. Hameyer and R. Belmans, Numerical Modelling and Design of Electrical Machines and Devices, WIT Press, Southampton, 1999.

[18] D.A. Lowther and P.P. Silvester, Computer-Aided Design in Magnetics, Springer, London, 1985.

[19] OPERA, Vector Fields Ltd, http://www.vectorfields.co.uk/.

[20] MagNet, Infolytica, http://www.infolytica.com/.

[21] Maxwell, Ansoft, http://www.ansoft.com/.

[22] ANSYS Multiphysics, http://www.ansys.com/products/default.asp.

[23] FLUX, CEDRAT Software, http://www.cedrat.com/.

[24] MEGA, http://www.bedl.co.uk/.

[25] Integrated Engineering Software, http://www.integratedsoft.com/.

[26] SPEED, http://www.speedlab.co.uk/software.html.

[27] C.W. Trowbridge and J.K. Sykulski, "Some key developments in computational electromagnetics and their attribution", IEEE Transactions on Magnetics 42 (4), 503-508 (2006).

[28] R. Southwell, Relaxation Methods in Theoretical Physics, OUP, Oxford, 1946.

[29] M.J. Turner et al, "Stiffness and deflection analysis of complex structures", J. Aero Sci. 23, 805-806 (1956).

[30] A.M. Winslow, "Numerical calculation of static magnetic fields in an irregular triangle mesh", J Comput Phys 1, 149150 (1966).

[31] P.P. Silvester, "High-order polynomial triangular finite elements for potential problems", Int. J. Engineering Science 7, 849-861 (1969).

[32] M.V.K. Chari and P.P. Silvester, "Finite element analysis of magnetically saturated dc machines", IEEE Trans. PAS 89 (7), 1642-51 (1970) and 90 (2), 454-464 (1971).

[33] J.A. Meijerink and V. der Vorst, "An iterative solution method for systems of which the coefficient matrix is a symmetric M matrix", Maths. Comp. 31, 148-149 (1977).

[34] J. Simkin and C.W. Trowbridge, "On the use of the total scalar potential in the numerical solution of field problems in electromagnetics", IJNME 14, 432-433 (1978).

[35] Z. Cendes, D. Shenton, and M. Shahnasser, "Magnetic field computation using Delaunay triangulation and complementary finite element methods", IEEE Trans. on Magnetics 19, 2551-2554 (1983).
[36] L. Janucke and A. Kost, "Error estimation and adaptive mesh generation in the 2D and 3D finite element method", IEEE Trans. Magn. 32 (3), (1992).

[37] E.M. Freeman and D.A. Lowther, "A novel mapping technique for open boundary finite element solutions to poissons equation”, IEEE Trans. Magn. 24 (6), (1988).

[38] J. Imhoff, G. Meunier and J.C. Sabonnadiere, "Finite element modelling of open boundary problems", IEEE Trans. Magn. 26 (2), (1990).

[39] A. Bossavit and J.C. Verite, "A mixed FEM-BIEM method to solve 3-D eddy current problem", IEEE Trans. Magn., 431435 (1982).

[40] A. Bossavit, "Whitney forms: a class of finite elements for three-dimensional computations in electromagnetism", IEE Proc. A 135, 493-500 (1988).

[41] O. Biro, K. Preis and K. Richter, "On the use of the magnetic vector potential in the nodal and edge finite element analysis of 3D magnetostatic problems", IEEE Trans. Magn. 32 (3), (1996).

[42] T. Yioultsis and T. Tsiboukis, "Multiparametric finite elements: a systematic approach to the construction of 3-D, higher order, tangential vector shape functions", IEEE Trans. Magn. 32 (3), (1996).

[43] D. Baldomir, "Differential forms and electromagnetism in 3dimensional Euclidian space $\mathrm{R}^{3}$ ", IEE Proc A 133, 139-140 (1986).

[44] Z. Ren, "Application of differential forms in the finite element formulation of electromagnetic problems", ICS Newsletter, 7 (3), 6-11 (2000).

[45] E. Tonti, "Finite formulation of electromagnetic field", ICS Newsletter, 8 (1), 5-11 (2001).

[46] P. Hammond and J. Penman, "Calculation of inductance and capacitance by means of dual energy principles", Proc IEE 123 (6), 554-559 (1976).

[47] J.K. Sykulski, "Computer package for calculating electric and magnetic fields exploiting dual energy bounds", IEE Proceedings A 135 (3), 145-150 (1988).

[48] I.M. Mayergoyz, "Mathematical models of hysteresis", IEEE Trans. Magn. 22, (1986).

[49] L. Dupre and J. Malkebeek, "Electromagnetic hysteresis modelling: from material science to finite element analysis of devices", ICS Newsletter, 10 (3), 4-14 (2003).

[50] A.G. Jack, B.C. Mecrow, P.G. Dickinson, and D. Stephenson, "Permanent-magnet machines with powdered iron cores and prepressed windings", IEEE Trans. Ind. Appl. 36, 1077-1084 (2000).

[51] J.K. Sykulski, K.F. Goddard, and R.L. Stoll, "A method of estimating the total AC loss in a high-temperature superconducting transformer winding", IEEE Trans. Magn. 36, 1183-7 (2000).

[52] I.O. Golosnoy and J.K. Sykulski, "Evaluation of the frontfixing method capabilities for numerical modelling of field diffusion in high-temperature superconducting tapes", IET Science, Measurement and Technology 2 (6), 418-426 (2008).

[53] A. Razek, J. Coulomb, M. Feliachi, and J.C. Sabonnadiere, "Conception of an Air-Gap Element for the Dynamic Analysis of the Electromagnetic Field in Electric machines", IEEE Trans. Magn. 18 (2), (1982).

[54] D. Rodger, H. Lai and P. Leonard, "Coupled elements for problems involving motion”, IEEE Trans. Magn. 26 (2), (1990). 
[55] I.A. Tsukerman, "Overlapping finite elements for problems with movement", IEEE Trans. Magn. 28 (5), 2247-2249 (1992).

[56] A. Demenko, "Movement simulation in finite element analysis of electric machine dynamics", IEEE Trans. Magn. 32 (3), (1996).

[57] C. Christopoulos, The Transmission-Line Modelling Method: TLM, IEEE Press and Oxford University Press, Oxford, 1995.

[58] P. Sewell, J.G. Wykes, T.M. Benson, D.W. Thomas, A. Vukovic, and C. Christopoulos, "Transmission line modelling using unstructured meshes", IEE Proc SMT 151 (6), 445-448 (2004).

[59] T. Weiland, "Time domain electromagnetic field computation with finite difference methods", Int. J. Numerical Modelling 9, 295-319 (1996).

[60] C.A. Brebbia and A. Kassab, Electrical Engineering and Electromagnetics VII, Wit Press, Southampton, 2006.

[61] Y. Takahashi and S. Wakao, "Large-scale analysis of eddycurrent problems by the hybrid finite element-boundary element method combined with the fast multipole method", IEEE Trans. Magn. 42 (4), 671-674 (2006).

[62] C.J. Carpenter, "Surface-integral methods of calculating forces on magnetized iron parts", IEE Monograph 342, 19-28 (1959).

[63] F. Henrotte, "Handbook for the computation of electromagnetic forces in a continuous medium", Int. Compumag Society Newsletter 24 (2), 3-9 (2004).

[64] J.L. Coulomb and G. Meunier, "Finite element implementation of virtual work principle for magnetic force and torque computation", IEEE Trans. Magn. 20, (1985).

[65] S. McFee, J. Webb, and D.A. Lowther, "A tunable volume integration formulation for force calculation in finite-element based computational magnetostatics", IEEE Trans. Magn. 24, 439-442 (1988).

[66] F. Henrotte, H.V. Sande, G. Deliege, and K. Hameyer, "Electromagnetic force density in a ferromagnetic material", IEEE Trans. Magn. 40, 553-556, (2004).

[67] D.H. Kim, D.A. Lowther, and J.K. Sykulski, "Efficient force calculations based on continuum sensitivity analysis", IEEE Trans. Magn. 41 (5), 1404-1407 (2005).

[68] D.H. Kim, D.A. Lowther, and J.K. Sykulski, "Efficient global and local force calculations based on continuum sensitivity analysis", IEEE Trans. Magn. 43 (4), 1177-1180 (2007).

[69] M. Li, D.H. Kim, D.A. Lowther, and J.K. Sykulski, "A sensitivity approach to force calculation in electrostatic MEMS devices", IEEE Transactions on Magnetics 44 (6), 1610-3 (2008).

[70] J.K. Sykulski, K. Goddard, and R.L. Stoll, "High temperature super-conducting demonstrator transformer: design considerations and first test results", IEEE Trans. on Magnetics 35 (5), 3559-61 (1999).

[71] J.K. Sykulski, C. Beduz, R.L. Stoll, M.R. Harris, K. Goddard, and Y. Yang, "High temperature superconducting power transformers: conclusions from a design study", IEE Proceedings; Electrical. Power Applications 146 (1), 41-52 (1999).

[72] M.K. Mosawi, C.Beduz, K.F. Goddard, J.K. Sykulski, Y. Yang, B. Xu, K.S. Ship, R. Stoll, and N.G. Stephen, "Design of a $100 \mathrm{kVA}$ high temperature superconducting demonstration synchronous generator", Physica C 372-6(P3), 1539 1542 (2002).

[73] K.F. Goddard, J.K. Sykulski, and R.L. Stoll, "A new approach to modelling dominant AC loss in HTc supercon- ducting solenoidal windings", IEEE Trans. on Magn. 35 (3), 1195-1198 (1999).

[74] J.K. Sykulski, M. Rotaru, and R.L. Stoll, "2D modelling of field diffusion and AC losses in high temperature superconducting tapes", IEEE Trans. on Magn. 36 (4), 1178-1182 (2000).

[75] B. Lukasik, K.F. Goddard, and J.K. Sykulski, "Finite-element assisted method to reduce harmonic content in the air-gap flux density of a high-temperature superconducting coreless rotor generator", IET Science, Measurement and Technology 2 (6), 485-492 (2008).

[76] K.F. Goddard, B. Lukasik, and J.K. Sykulski, "Alternative designs of a superconducting synchronous generator: the Southampton approach", Int. Conf. Electrical Machines ICEM, (CD-ROM), (2008).

[77] A. Demenko and J.K. Sykulski, "Network equivalents of nodal and edge elements in electromagnetics", IEEE Transactions on Magnetics 38 (2), 1305-1308 (2002).

[78] A. Demenko and J.K. Sykulski, "Magneto-electric network models in electromagnetism", COMPEL: The International J. Computation and Mathematics in Electrical and Electronic Engineering 25 (3), 581-588 (2006).

[79] A. Demenko, J.K. Sykulski, and R. Wojciechowski, "Network Representation of Conducting Regions in 3-D Finite-Element Description of Electrical Machines", IEEE Transactions on Magnetics 44 (6), 714-717 (2008).

[80] A. Demenko, J.K. Sykulski, and R. Wojciechowski, "Calculation of inducted currents using edge elements and T-T $T_{0}$ formulation", IET Sci. Meas. Technol. 2 (6), 434-439 (2008).

[81] A. Sobester, S.J. Leary, and A.J. Keane, "On the design of optimization strategies based on global response surface approximation Models", J. Global Optimization 33, 31-59 (2005).

[82] M. Farina and J.K. Sykulski, "Comparitive study of evolution strategies combined with approximation techniques for practical electromagnetic optimization problems", IEEE Trans. Magnetics 37 (5), 3216-3220 (2001).

[83] L. Lebensztajn, C.A.R. Marretto, M.C. Costa, and J-L. Coulomb, "Kriging: a useful tool for electromagnetic devices optimization", IEEE Trans. Magnetics 40 (2), 1196-1199 (2004).

[84] D.H. Kim, K.F. Ship and J.K. Sykulski, "Applying continuum design sensitivity analysis combined with standard EM software to shape optimisation in magnetostatic problems", IEEE Trans. on Magnetics 40 (2), 1156-1159 (2004).

[85] D.H. Kim, J.K. Sykulski and D. Lowther, "A novel scheme for material updating in source distribution optimization of magnetic devices using sensitivity analysis", IEEE Trans. on Magnetics 41 (5), 1752-1755 (2005).

[86] D.H. Kim, D.A. Lowther, and J.K. Sykulski, "Smooth boundary topology optimisation applied to an electrostatic actuator", IET Science, Measurement and Technology 2 (6), 427433 (2008).

[87] J.K. Sykulski, "New trends in optimization in electromagnetics", Przeglad Elektrotechniczny 83 (6), 13-18 (2007).

[88] D.H. Wolpert and W.G. Macready, "No free lunch theorems for optimization", IEEE Trans. Evol. Comp. 1 (1), 67-82 (1997).

[89] W.P. Baritompa, M. Dur, E.M.T. Hendrix, L. Noakes, W.J. Pullan and G.R. Wood, "Matching stochastic algorithms to objective function landscapes", J. Global Optimization 31 (4), 579-598 (2005). 
[90] D.R. Jones, "A taxonomy of global optimization methods based on response surfaces", J. Global Optimization 21, 345374 (2001).

[91] A.J. Keane, "Statistical improvement criteria for use in multiobjective design optimization", AIAA Journal 44 (4), 879891 (2006).

[92] T.J. Santner, B.J. Williams, and W.I. Notz, The Design and Analysis of Computer Experiments, Springer, London, 2003.

[93] D.R. Jones, M. Schonlau, and W.J. Welch, "Efficient global optimization of expensive black-box functions", J. Global Optimization 13, 455-492 (1998).

[94] A. Sobester, S.J. Leary, and A.J. Keane, "On the design of optimization strategies based on global response surface approximation models", J. Global Optimization 33, 31-59 (2005).

[95] G.I. Hawe and J.K. Sykulski, "A scalarizing one-stage algorithm for efficient multi-objective optimization", IEEE Transactions on Magnetics 44 (6), 1094-1097 (2008).

[96] K.M. Miettinen, Nonlinear Multiobjective Optimization, Kluwer Academic Publishers, London, 1999.

[97] J. Knowles, "ParEGO: A hybrid algorithm with on-line landscape approximation for expensive multiobjective optimization problems", IEEE Trans. Evol. Comp. 10 (1), 50-66 (2006).

[98] L. Lebensztajn, et al, "A multi-objective analysis of a special switched reluctance motor", COMPEL 24 (3), 931941 (2005).
[99] A.J. Keane, "Statistical improvement criteria for use in multiobjective design optimization", AIAA Journal 44 (4), 879891 (2006).

[100] M.T.M. Emmerich, K.C. Giannakoglou, and B. Naujoks, "Single- and multiobjective evolutionary optimization assisted by gaussian random field metamodels", IEEE Trans. Evol. Comp. 10 (4), 421-439 (2006).

[101] D. Montgomery, Design and Analysis of Experiments, John Wiley and Sons, New York, 2001.

[102] J.R. Kalagnanam and U.M. Diwekar, "An efficient sampling technique for off-line quality control”, Technometrics 39 (3), 308-318 (1997).

[103] 13th Biennial IEEE Conference on Electromagnetic Field Computation CEFC 2008, Athens, Greece, 11-15 May 2008, http://www.cefc2008.gr/cefc2008/index.php, (2008).

[104] 14th International Symposium on Electromagnetic Fields in Electrical and Electronic Engineering (ISEF), Arras, France, 10-12 September 2009. http://www.lsee.fr/isef09/, (2009).

[105] International Conference on Electrical Machines, Vilamoura, Portugal Greece, 6-9 Sept 2008, http://www.apdee.org/index.php?pageid=1237, (2008).

[106] IGTE Symposium on Numerical Field Calculation in Electrical Engineering, Graz University of Technology, Austria, 2124 September, 2008, http://www.igte.tugraz.at/symp08/cms/, (2008).

[107] COMPUMAG 2009, Florianopolis, Brazil, 22-26 November 2009, http://www.grucad.ufsc.br/c2009/, (2009). 